\title{
Isotropy and Marginally Trapped Surfaces in a Spacetime
}

\author{
J. L. Cabrerizo ${ }^{1}$, M. Fernández and J. S. Gómez \\ Geometría y Topología, Universidad de Sevilla, Apdo. 1160, 41080 Sevilla, Spain \\ e-mails: jaraiz@us.es, mafernan@us.es, jsalvador@us.es
}

\begin{abstract}
In this note we shall study the notions of isotropic and marginally trapped surface in a spacetime by using a differential geometric approach. We first consider spacelike isotropic surfaces in a Lorentzian manifold and, in particular, in a four-dimensional spacetime, where the isotropy function appears to be determined by the mean curvature vector field of the surface. Explicit examples of isotropic marginally outer trapped surfaces are given in the standard fourdimensional space forms: Minkowski, De Sitter and anti De Sitter spaces. Then we prove ridigity theorems for complete spacelike 0-isotropic surfaces without flat points in these standard space forms. As a consequence, we also obtain characterizations of complete spacelike isotropic marginally trapped surfaces in these backgrounds.
\end{abstract}

PACS numbers: 04.20.Cv, 02.40.Ky, 02.40.Ma

\section{Introduction}

A precise definition of isotropy depends on the subject area. In Mathematics, a Riemannian manifold is isotropic if, roughly speaking, the geometry of the manifold is the same regardless of direction. The concept of isotropic immersion of a Riemannian manifold $S$ in another $M$ was introduced by B. O'Neill in 1965 [1] as an isometric immersion such that all its normal curvature vectors have the same length. These submanifolds can be considered as a generalization of the totally umbilical submanifolds, and constitute a distinguished family in Submanifold Theory. Later, Y. H. Kim [2] introduced the notion of pseudo-isotropic submanifold by extending the O'Neill's notion to the case of pseudo-Riemannian submanifolds of pseudo-Euclidean space. Since both notions are basically identical, from now on we shall omit the prefix pseudo and call them all isotropic submanifolds. However, new proof methods are usually required to achive similar results to those obtained in the Riemannian case $[3,4]$.

On the other hand, in studies of gravitational collapse, an useful local property is the closed trapped surface, introduced by R. Penrose in 1965 [5]. This is a closed

\footnotetext{
${ }^{1}$ Author to whom the correspondence should be directed
} 
spacelike two-dimensional surface $S$ at any instant of time which has the property that the outgoing light rays from it are convergent. In the spherical symmetric case the existence of a trapped surface garantees that the system must collapse to a black hole assuming only that the matter has positive energy density (the week energy condition). The idea that matter concentration causes gravitational collapse is the trapped surface conjecture: a trapped surface forms if a sufficient amount of matter is packed into a small enough volume. In [6] the authors give necessary and sufficient conditions for the existence of trapped surfaces in sphericaly symmetric spacetimes, and in [7] the authors by using the spin coefficient formalism generalize from the Schwarzschild solution and prove the existence of a wide class of solutions possessing trapped surfaces, includding the so-called "asymptotically trapped surfaces". In [8] the non-existence of these closed surfaces is proved for strictly stationary spacetimes. In it is shown that the existence of one trapped surface in a spherical symmetric spacetime is sufficient to ensure the formation of a black hole and the completeness of null infinity. A generalization of the concept of trapped surface is introduced in [9] for codimension-2 submanifolds of a Lorentzian space.

From a geometrical point of view, the light converging condition means that the mean curvature vector $\mathbf{H}$ is everywhere timelike on the surface. If the mean curvature vector is future or past-pointing all over the surface, the trapped surface is accordingly called future or past trapped. On the other hand, consider two futurepointing lightlike normal vectors at each point of the surface $S$ and suppose the mean curvature vector $\mathbf{H}$ is proportional to some of them by a function $\Theta$. The following cases may occurs: (1) $S$ is marginally trapped if $\Theta$ never vanishes; (2) partly marginally trapped if $\Theta \geq 0$ or $\Theta \leq 0$; (3) marginally outer trapped (MOTS) if $\Theta$ is arbitrary. The study of these family of surfaces has been quite active in recent years (see for instance $[9,10,11,12,13]$, etc).

It is clear that the second fundamental form of $S$ is a suitable tool to study these families of surfaces. Thus, a classification of spacelike marginally trapped surfaces in the Lorentzian space forms $\mathbb{R}_{1}^{n}, \mathbb{S}_{1}^{n}$ and $\mathbb{H}_{1}^{n}$ having the property that the second fundamental form is a quadratic degenerated form (in the sense that the surfaces have positive relative nullity) have been obtained in [14]. The same authors proved the nonexistence of marginally trapped surfaces with positive relative nullity for a Robertson-Walker spacetime which does not contain any open subset of constant curvature [15].

The plan of the paper is as follows. Basic formulas, definitions and facts on $n$-dimensional Lorentz manifolds $M_{1}^{n}$ are introduced in Section II. In Section III we first recall the notion of isotropic surface $S$ in $M_{1}^{n}$ and, as a consequence, we see that it is pseudo-umbilical, but for $n=3$ the surface is totally umbilical. Section IV deals with the interesting case of a surface embedded into a 4-dimensional spacetime $M_{1}^{4}$, where the notions of $\lambda$-isotropy and pseudo-umbilicity for surfaces with lightlike mean curvature vector are seen to be equivalent. Some properties of the isotropy function $\lambda$ are obtained, in particular, characterizations of 0 -isotropic surfaces. In Section V we first exhibit examples of 0-isotropic MOTS in the 4dimensional spacetimes $\mathbb{R}_{1}^{4}, \mathbb{S}_{1}^{4}$ and $\mathbb{H}_{1}^{4}$, and then we give sufficient conditions for 
them to be non-totally umbilical marginally trapped surfaces. Finally, we present our main results that provide ridigity theorems, that is, explicit determination of complete spacelike 0 -isotropic surfaces without flat points in these spacetimes. In fact, they are seen to be MOTS congruent to the corresponding immersed surfaces introduced as Examples 5.1, 5.2 and 5.3. As a consequence, we obtain a characterization of complete spacelike isotropic marginally trapped surfaces as well as complete pseudo-umbilical marginally trapped surfaces in these standard space forms. We close the paper with a summary of conclusions.

\section{Preliminaries}

Let $M_{1}^{n}$ be a $n$-dimensional Lorentzian manifold with metric tensor $g$. All the manifolds will be assumed to be connected without boundary. Let $\phi: S \rightarrow M_{1}^{n}$ be an isometric immersion of a 2-dimensional spacelike surface $S$. For all local formulae and computations we may assume $\phi$ is an imbedding and thus we shall often identify $p \in S$ with $\phi(p) \in M_{1}^{n}$, and the tangent space $T_{p} S$ with the subspace $\phi_{*}\left(T_{p} S\right)$ of $T_{p} M_{1}^{n}$. The normal bundle will be denoted by $T^{\perp} S$. We shall use letters $X, Y, Z$ (resp. $\xi, \eta, \zeta$ ) to denote vectors fields tangent (resp. normal) to $S$. A tangent vector $v \in T_{p} M_{1}^{n}$ is said to be spacelike (timelike or lightlike) if $g(v, v) \geq 0(g(v, v)<0$, $g(v, v)=0$ and $v \neq 0$, respectively). Let $\tilde{\nabla}$ and $\nabla$ be the Levi-Civita connections of $M_{1}^{n}$ and $S$, respectively. Then, the Gauss-Weingarten formulas are given by

$$
\begin{aligned}
\tilde{\nabla}_{X} Y & =\nabla_{X} Y+h(X, Y) \\
\tilde{\nabla}_{X} \xi & =-A_{\xi} X+D_{X} \xi
\end{aligned}
$$

where $h$ denotes the second fundamental form of $\phi, A_{\xi}$ the shape operator, and $D$ is the normal connection. The shape operator and the second fundamental form are related by $g\left(A_{\xi} X, Y\right)=g(h(X, Y), \xi)$.

Denote by $\tilde{R}$ and $R$ the curvature tensor of $M_{1}^{n}$ and $S$, respectively. If $M_{1}^{n}$ is of constant sectional curvature $c$, then $\tilde{R}$ is given by $\tilde{R}(X, Y) Z=c\{g(Y, Z) X-$ $g(X, Z) Y\}$. The curvature tensor $R$ is given by $R(X, Y) Z=\mathcal{K}\{g(Y, Z) X-g(X, Z) Y\}$, where $\mathcal{K}$ is the Gauss curvature of $S$.

If $p \in S$ and $v \in T_{p} M_{1}^{n}$ we shall denote by $v^{\top}$ and $v^{\perp}$ the tangential and normal components of $v$, respectively. The equations of Gauss and Codazzi are given, respectively, by

$$
\begin{aligned}
(\tilde{R}(X, Y) Z)^{\top} & =R(X, Y) Z+A_{h(X, Z)} Y-A_{h(Y, Z)} X, \\
(\tilde{R}(X, Y) Z)^{\perp} & =\left(\bar{\nabla}_{X} h\right)(Y, Z)-\left(\bar{\nabla}_{Y} h\right)(X, Z),
\end{aligned}
$$

where $\bar{\nabla} h$ is defined by

$$
\left(\bar{\nabla}_{X} h\right)(Y, Z)=D_{X}(h(Y, Z))-h\left(\nabla_{X} Y, Z\right)-h\left(Y, \nabla_{X} Z\right) .
$$


If $p \in S$ and $x, y \in T_{p} S$ are linearly independent tangent vectors, we define the discriminant $\mathcal{D}_{p}$ at $p$ as

$$
\mathcal{D}_{p}=\frac{g(h(x, x), h(y, y))-g(h(x, y), h(x, y))}{g(x, x) g(y, y)-g(x, y)^{2}} .
$$

If $\tilde{\mathcal{K}}$ denotes the sectional curvature of $M_{1}^{n}$, from Eq. (2.3) we easily have

$$
\widetilde{\mathcal{K}}(x, y)=\mathcal{K}_{p}-\mathcal{D}_{p}
$$

Note that if $\mathcal{D} \equiv 0$ then $S$ is extrinsically flat: observers in $M_{1}^{n}$ see no curving. This does not mean that $S$ is intrinsically flat, but the surface $S$ has the same intrinsic curvature as $M_{1}^{n}$.

Recall that a point $p \in S$ is called umbilic [16] provided there exists a vector $\xi_{p} \in T_{p}^{\perp} S$ such that $h(u, v)=g(u, v) \xi_{p}$ for any $u, v \in T_{p} S$. The immersion $\phi$ is said to be totally umbilical when every point of $S$ is umbilic. In such a case, it is easy to see that $h(X, Y)=g(X, Y) \mathbf{H}$ for any $X, Y \in \mathfrak{X}(S)$, where $\mathbf{H}=(1 / 2)$ trace $_{g}(h)$ is the mean curvature vector of $S$. We also recall that a point $p \in S$ is said to be flat if $h=0$ at $p$, and the immersion $\phi$ is totally geodesic provided every point of $S$ is flat.

The immersion $\phi$ is called pseudo-umbilical if its second fundamental form $h$ satisfies $g(h(X, Y), \mathbf{H})=\rho g(X, Y)$ for some function $\rho$. Necessarily this function $\rho$ is given by $\rho=g(\mathbf{H}, \mathbf{H})$. Clearly, any totally umbilical immersion is pseudoumbilical.

The first normal space of $\phi$ at $p \in S$ is defined as the subspace spanned by the second fundamental form $h$ at $p$, that is,

$$
\operatorname{Im}(h)_{p}=\operatorname{span}\left\{h(u, v): u, v \in T_{p} S\right\} .
$$

A simple computation shows that $\left\{\xi \in T_{p}^{\perp} S: A_{\xi}=0\right\}$ is the orthogonal complement of $\operatorname{Im}(h)_{p}$ in $T_{p}^{\perp} S$. Observe that if the dimension of $\operatorname{Im}(h)$ is constant along $S$, then $\operatorname{Im}(h)$ is a subbundle of $T^{\perp} S$.

\section{Isotropic immersion}

We first recall that an isometric immersion $\phi: S \rightarrow M_{1}^{n}$ is called isotropic at $p \in S$ [2] if

$$
g(h(u, u), h(u, u))=\lambda(p) \in \mathbb{R}
$$

does not depends on the choice of the unit tangent vector $u \in T_{p} S$, and $\phi$ is said to be isotropic if $\phi$ is isotropic at each point of $S$. In such a case, the smooth function $\lambda: S \rightarrow \mathbb{R}$ defined by equation (3.1) is called the isotropy function, and the isometric immersion $\phi$ is said to be $\lambda$-isotropic. In particular, if $\lambda$ is a constant function, then $\phi$ is said to be a constant isotropic immersion.

Remark 3.1 It is clear that every totally umbilical immersion $\phi: S \rightarrow M_{1}^{n}$ is an isotropic immersion with isotropy function $\lambda=g(\mathbf{H}, \mathbf{H})$, but not vice versa. Notice also that if $\phi$ is totally umbilical and $M_{1}^{n}$ has constant sectional curvature $c$, then $\mathbf{H}$ is a parallel vector field and the function $g(\mathbf{H}, \mathbf{H})$ is a constant [17, p.11]. 
Similar to the Riemannian case [1], it is easy to prove the following.

Lemma 3.2 Let $\phi: S \rightarrow M_{1}^{n}$ be a spacelike isometric immersion. Then, the following conditions are equivalent:

(1) $\phi$ is isotropic at $p$.

(2) $g(h(x, y), h(z, w))+g(h(y, z), h(x, w))+g(h(z, x), h(y, w))=\lambda(p)\{g(x, y) g(z, w)+$ $g(y, z) g(x, w)+g(x, z) g(y, w)\}$ for all $x, y, z, w \in T_{p} S$.

Furthermore, if $\phi$ is isotropic at $p$ we have

$$
\begin{aligned}
& g(h(u, u), h(u, v))=0, \\
& g(h(u, u), h(v, v))+2 g(h(u, v), h(u, v))=\lambda(p),
\end{aligned}
$$

for any orthonormal tangent vectors $u, v \in T_{p} S$.

Remark 3.3 As a consequence of equation (3.2) we see that an isotropic immersion of a spacelike surface $S$ into a 3 -dimensional Lorentzian manifold $M_{1}^{3}$ is totally umbilical, since $T^{\perp} S$ is a 1-dimensional negative defined space [4].

Lemma 3.4 Let $\phi: S \rightarrow M_{1}^{n}$ be a spacelike $\lambda$-isotropic immersion. Then, $\phi$ is pseudo-umbilical and $\mathbf{H}, \lambda$ and $\mathcal{D}$ satisfy the equation

$$
3 g(\mathbf{H}, \mathbf{H})=2 \lambda+\mathcal{D} .
$$

PROOF. Let $\left\{e_{1}, e_{2}\right\}$ be a local orthonormal frame tangent to $S$. Since $\mathbf{H}=$ $\left(h\left(e_{1}, e_{1}\right)+h\left(e_{2}, e_{2}\right)\right) / 2$, equations $(2.5)$ and $(3.3)$ give $\mathcal{D}=\lambda-3 g\left(h\left(e_{1}, e_{2}\right), h\left(e_{1}, e_{2}\right)\right)$. Thus $g\left(h\left(e_{1}, e_{1}\right), \mathbf{H}\right)=g\left(h\left(e_{2}, e_{2}\right), \mathbf{H}\right)=(2 \lambda+\mathcal{D}) / 3$. On the other hand, Eq. (3.2) yields $g\left(h\left(e_{1}, e_{2}\right), \mathbf{H}\right)=0$, and by linearity $g(h(X, Y), \mathbf{H})=((2 \lambda+\mathcal{D}) / 3) g(X, Y)$ for any $X, Y \in \mathfrak{X}(S)$. This means that $S$ is pseudo-umbilical with associated function $\rho=g(\mathbf{H}, \mathbf{H})=(2 \lambda+\mathcal{D}) / 3$.

\section{Isotropic surfaces in 4-dimensional spacetimes}

When the mean curvature vector field $\mathbf{H}$ of an immersed spacelike surface in a spacetime $M_{1}^{n}$ is a lightlike vector field and $n=4$, then the notions of isotropy and pseudo-umbilicity are equivalent. In fact, we have the following.

Proposition 4.1 Let $\phi: S \rightarrow M_{1}^{4}$ be a spacelike surface with lightlike mean curvature vector. Then, the following assertions are equivalent.

(1) $\phi$ is pseudo-umbilical.

(2) $\phi$ is 0-isotropic. 
(3) $\phi$ is isotropic.

PROOF. (1) $\Rightarrow$ (2). Since $g(h(X, X), \mathbf{H})=0$ and the normal space is a Lorentzian plane we have $g(h(X, X), h(X, X))=0$. Now (2) $\Rightarrow$ (3) is obvious, and (3) $\Rightarrow$ (1) follows from Lemma 3.4.

Theorem 4.2 Let $S$ be a spacelike $\lambda$-isotropic surface in a spacetime $M_{1}^{4}$. Then, we have

(a) If $p \in S$ is not an umbilical point, then $\lambda(p)=g(\mathbf{H}, \mathbf{H})_{p}=\mathcal{D}_{p}=0$.

(b) The isotropy function $\lambda$ is given by $\lambda=g(\mathbf{H}, \mathbf{H})=\mathcal{D}$.

(c) For any $X, Y, Z, W \in \mathfrak{X}(S)$ we have

$$
g(h(X, Y), h(Z, W))=\lambda g(X, Y) g(Z, W) .
$$

PROOF. (a) Assume $p \in S$ is not an umbilical point. Take $e_{1}, e_{2} \in T_{p} S$ orthonormal vectors such that $h\left(e_{1}, e_{2}\right) \neq 0$. Since $\phi$ is $\lambda$-isotropic then

$$
g\left(h\left(e_{i}, e_{i}\right), h\left(e_{i}, e_{i}\right)\right)=\lambda(p)
$$

for $i=1,2$. Now we shall prove $\lambda(p)=0$.

Case (i). Suppose $\lambda(p)>0$. Equation (3.2) yields $g\left(h\left(e_{1}, e_{1}\right), h\left(e_{1}, e_{2}\right)\right)=0$, and we have that $h\left(e_{1}, e_{1}\right) \neq 0$ is spacelike and $h\left(e_{1}, e_{2}\right)$ is timelike. Let us write

$$
\begin{aligned}
& h\left(e_{1}, e_{1}\right)=\delta e_{3}, \\
& h\left(e_{1}, e_{2}\right)=\mu e_{4},
\end{aligned}
$$

where $\left\{e_{1}, e_{2}, e_{3}, e_{4}\right\}$ is an orthonormal frame of $T_{p} M_{1}^{4} \cong \mathbb{R}_{1}^{4}\left(e_{4}\right.$ is a unit timelike vector $)$, and $\delta=[\lambda(p)]^{1 / 2}>0, \mu=\left[-g\left(h\left(e_{1}, e_{2}\right), h\left(e_{1}, e_{2}\right)\right)\right]^{1 / 2}>0$. On the other hand, from Eq. (3.2) we have also that $g\left(h\left(e_{1}, e_{2}\right), h\left(e_{2}, e_{2}\right)\right)=0$. Therefore, equations (4.2) and (4.4) give $h\left(e_{2}, e_{2}\right)= \pm \delta e_{3}$. If $h\left(e_{2}, e_{2}\right)=\delta e_{3}$, then from Eq. (3.3) we have

$$
g\left(h\left(e_{1}, e_{1}\right), h\left(e_{2}, e_{2}\right)\right)+2 g\left(h\left(e_{1}, e_{2}\right), h\left(e_{1}, e_{2}\right)\right)=\lambda(p) .
$$

Thus equations (4.3) and (4.4) yield $g\left(h\left(e_{1}, e_{2}\right), h\left(e_{1}, e_{2}\right)\right)=0$, which is a contradiction because $\mu>0$. The case $h\left(e_{2}, e_{2}\right)=-\delta e_{3}$ also gives a contradiction with $\mu^{2}=-\lambda(p)$.

Case (ii). Assume $\lambda(p)<0$. As in Case (i), if we write $h\left(e_{1}, e_{1}\right)=\delta e_{4}, h\left(e_{1}, e_{2}\right)=$ $\mu e_{3}$, with $\delta=[-\lambda(p)]^{1 / 2}$ and $\mu=\left[g\left(h\left(e_{1}, e_{2}\right), h\left(e_{1}, e_{2}\right)\right)\right]^{1 / 2}$ we get a contradiction.

Finally, using equations (2.5) and (3.4), we have $\lambda(p)=g(\mathbf{H}, \mathbf{H})_{p}=\mathcal{D}_{p}=0$.

(b) From Remark 3.1, at any umbilical point we have $\lambda=g(\mathbf{H}, \mathbf{H})$, and then Eq. (3.4) gives $\lambda=\mathcal{D}=g(\mathbf{H}, \mathbf{H})$. Therefore, in a non-umbilical point the result follows from assertion (a). 
(c) For any $p \in S$ and $x, y, z, w \in T_{p} S$ define the multilinear function $F$ on $T_{p} S$ as follows.

$$
\begin{aligned}
F(x, y, z, w)= & g(h(z, x), h(y, w))-g(h(y, z), h(x, w)) \\
& -\lambda(p)\{g(z, x) g(y, w)-g(y, z) g(x, w)\} .
\end{aligned}
$$

Then, $F$ is a curvaturelike function, i.e., $F$ has the following symmetries:

(i) $F(x, y, z, w)+F(y, z, x, w)+F(z, x, y, w)=0$,

(ii) $F(y, x, z, w)=-F(x, y, z, w)$,

(iii) $F(x, y, w, z)=-F(x, y, z, w)$,

(iv) $F(z, w, x, y)=F(x, y, z, w)$,

But Eq. (2.5) yields $F(x, y, y, x)=0$ for any $x, y \in T_{p} S$ such that $Q(x, y)=$ $g(x, x) g(y, y)-g(x, y)^{2} \neq 0$, and therefore [16] $F=0$. In particular,

$$
\begin{aligned}
g(h(z, x), h(y, w))-\lambda(p) g(z, x) g(y, w) & =g(h(y, z), h(x, w))-\lambda(p) g(y, z) g(x, w)= \\
& =g(h(x, y), h(z, w))-\lambda(p) g(x, y) g(z, w) .
\end{aligned}
$$

Now, using (2) of Lemma 3.2, Eq. (4.1) follows.

Remark 4.3 Observe that an isotropic surface into a Riemannian manifold which satisfies Eq. (4.1) is totally umbilical. The Lorentzian version, however, is not true.

As a immediate consequence we have the following two corollaries.

Corollary 4.4 Let $\phi: S \rightarrow M_{1}^{4}$ be a spacelike isotropic immersion. Then, the following assertions are equivalent.

(1) $\phi$ is 0-isotropic.

(2) $g(h(X, Y), h(Z, W))=0$ for any $X, Y, Z, W \in \mathfrak{X}(S)$.

(3) $g(\mathbf{H}, \mathbf{H})=0$.

(4) $S$ is extrinsically flat, i.e., the discriminant $\mathcal{D} \equiv 0$.

(5) The first normal space $\operatorname{Im}(h)$ is a lightlike line at each non-flat point.

Corollary 4.5 Let $\phi: S \rightarrow M_{1}^{4}$ be a spacelike isotropic immersion without umbilical points. Then, $\phi$ is 0-isotropic.

Corollary 4.6 Let $\phi: S \rightarrow M_{1}^{4}$ be a non-totally umbilical spacelike constant isotropic immersion. Then, $\phi$ is 0-isotropic. 
PROOF. Set $\mathcal{U}=\left\{p \in S: h(u, v) \neq 0\right.$ for some orthonormal vectors $\left.u, v \in T_{p} S\right\}$. Note that $\mathcal{U}$ is a non-empty open set which is the set of not umbilical points of $S$. From Theorem 4.2 the isotropy function $\lambda$ satisfies $\left.\lambda\right|_{\mathcal{U}}=\left.g(\mathbf{H}, \mathbf{H})\right|_{\mathcal{U}}=0$. Now, let $p \in \overline{\mathcal{U}}$ be an umbilical point in the boundary of $\mathcal{U}$ and take a sequence $\left\{p_{N}\right\}_{N \in \mathbb{N}}$ in $S$ which converges to $p$ and such that every $p_{N}$ is not umbilical. Let $X$ be a local unitary vector field around $p$ and consider the function $\lambda=g(h(X, X), h(X, X))$. We have

$$
\lambda(p)=\lim _{N} g\left(h\left(X\left(p_{N}\right), X\left(p_{N}\right)\right), h\left(X\left(p_{N}\right), X\left(p_{N}\right)\right)\right)=\lim _{N} \lambda\left(p_{N}\right)=0 .
$$

If $\mathcal{U}$ is dense the result follows. If $\mathcal{U}$ is not dense, the set $S-\overline{\mathcal{U}}$ is an open submanifold of $S$ which is totally umbilical, and thus constant $g(\mathbf{H}, \mathbf{H})$-isotropic. Now we take a sequence of umbilical points converging to a point in the boundary of $\mathcal{U}$, we obtain $\lambda=g(\mathbf{H}, \mathbf{H})=0$.

The following result can also be proved in a similar way as Corollary 4.6.

Corollary 4.7 Let $S$ be a non-totally umbilical spacelike isotropic surface of a spacetime $M_{1}^{4}(c)$ of constant sectional curvature $c$. Then, $\phi$ is 0-isotropic.

\section{Isotropic surfaces in a Lorentzian space form}

Let $\mathbb{R}_{s}^{n}$ be the $n$-dimensional pseudo-Euclidean space provided with the usual inner product of signature $s$ defined by

$$
\langle x, y\rangle=-\sum_{i=1}^{s} x_{i} y_{i}+\sum_{i=s+1}^{n} x_{i} y_{i},
$$

where $x=\left(x_{1}, \ldots, x_{n}\right), y=\left(y_{1}, \ldots, y_{n}\right)$. For a positive number $c$, the standard space form $\mathbb{S}_{s}^{n}(c)$ is the hypersurface $\mathbb{S}_{s}^{n}(c)=\left\{x \in \mathbb{R}_{s}^{n+1}:\langle x, x\rangle=1 / c\right\}$ endowed with the induced metric of signature $s$. For a negative number $c$, the standard space form $\mathbb{H}_{s}^{n}(c)$ is the hypersurface $\mathbb{H}_{s}^{n}(c)=\left\{x \in \mathbb{R}_{s+1}^{n+1}:\langle x, x\rangle=1 / c\right\}$ endowed with the induced metric of signature $s$. We simply denote $\mathbb{S}_{s}^{n}(1)$, and $\mathbb{H}_{s}^{n}(-1)$ by $\mathbb{S}_{s}^{n}, \mathbb{H}_{s}^{n}$, and the manifolds $\mathbb{S}_{s}^{n}, \mathbb{H}_{s}^{n}, \mathbb{R}_{1}^{n}$, are known as the Lorentzian spaces form models of constant curvature $c=1, c=-1$, and $c=0$, respectively. In general relativity, $\mathbb{R}_{1}^{4}$, $\mathbb{S}_{1}^{4}$ and $\mathbb{H}_{1}^{4}$ are called respectively as the Minkowski, de Sitter, and anti-de Sitter spacetimes.

It is well known that for every point $p \in \mathbb{S}_{1}^{n}\left(\right.$ resp. $\left.\mathbb{H}_{1}^{n}\right)$, any complete connected totally geodesic hypersurface of $\mathbb{S}_{1}^{n}$ (resp. $\mathbb{H}_{1}^{n}$ ) through $p$ is the connected component of the intersection of $\mathbb{S}_{1}^{n}$ (resp. $\mathbb{H}_{1}^{n}$ ) with a $n$-dimensional vector subspace $V$ of $\mathbb{R}_{1}^{n+1}$ (resp. $\mathbb{R}_{2}^{n+1}$ ) which contains $p$, and vice versa $[16, \mathrm{p} .105]$. These totally geodesic hypersurfaces are degenerates if and only if the $n$-dimensional vector subspace $V$ is degenerate, or equivalently, if the orthogonal complement of $V$ is a lightlike line.

We now show an example of an isotropic and marginally trapped surface in $\mathbb{R}_{1}^{4}$, which is not totally umbilical. 
Example 5.1 Let $f: \mathbb{R}^{2} \rightarrow \mathbb{R}$ be a smooth function. The isometric immersion $\psi: \mathbb{R}^{2} \rightarrow \mathbb{R}_{1}^{4}$ defined by

$$
\psi(x, y)=(f(x, y), x, y, f(x, y))
$$

is a 0-isotropic MOTS. In fact, it is easy to see that the second fundamental form $h$ and the mean curvature vector $\mathbf{H}$ are given respectively by

$$
\begin{aligned}
h(X, Y) & =\nabla^{2} f(X, Y) \eta, \\
\mathbf{H} & =\frac{1}{2} \Delta f \eta,
\end{aligned}
$$

where $\eta$ is the lightlike vector $(1,0,0,1) \in \mathbb{R}_{1}^{4}, \nabla^{2} f$ denotes the Hessian of $f$ in $\mathbb{R}^{2}$ and $\Delta f=\operatorname{trace}_{g}\left(\nabla^{2} f\right)$ is the corresponding Laplacian. Moreover, $\mathbf{H}$ is lightlike if and only if $\Delta f$ is nowhere zero, and $\psi$ is totally umbilical if and only if $f$ is given by

$$
f(x, y)=a\left(x^{2}+y^{2}\right)+b x+c y+d,
$$

with $a, b, c, d \in \mathbb{R}$. Notice that $\psi\left(\mathbb{R}^{2}\right)$ is contained in a degenerate hyperplane $x_{1}=x_{4}$ (in the natural coordinates of $\mathbb{R}_{1}^{4}$ ). Obviously, there exist functions $f \in C^{\infty}\left(\mathbb{R}^{2}\right)$ such that $\mathbf{H}$ is a non-parallel lightlike vector field. In such cases $\mathbb{R}^{2}$ is a non-totally umbilical isotropic marginally trapped surface embedded in $\mathbb{R}_{1}^{4}$.

In the next example we show an isotropic marginally trapped immersion of $\mathbb{S}^{2}$ into $\mathbb{S}_{1}^{4}$ which is non-totally umbilical.

Example 5.2 Let $f: \mathbb{S}^{2} \rightarrow \mathbb{R}$ be a smooth function. The isometric immersion $\psi: \mathbb{S}^{2} \rightarrow \mathbb{S}_{1}^{4}$ defined by

$$
\psi(x, y, z)=(f(x, y, z), x, y, z, f(x, y, z)),
$$

where $(x, y, z)$ denote the canonical coordinates of $\mathbb{R}^{3}$, is a 0 -isotropic MOTS in the Lorentzian space $\mathbb{S}_{1}^{4}$. In fact, let $\nabla^{2} f$ be the Hessian of the function $f$ in the sphere $\mathbb{S}^{2}$. A straightforward computation shows [18] that the second fundamental form $h$ of $\psi$ is given by

$$
h(X, Y)=\left(\left(\nabla^{2} f\right)(X, Y)+f g(X, Y)\right) \eta,
$$

where $\eta$ denote the lightlike vector $(1,0,0,0,1) \in \mathbb{R}_{1}^{5}$. Thus, the immersion $\psi$ is 0 -isotropic with mean curvature vector

$$
\mathbf{H}=\frac{1}{2}(\Delta f+2 f) \eta
$$

Note that $g(\psi, \eta)=0$ means that $\psi\left(\mathbb{S}^{2}\right)$ is contained in the degenerate totally geodesic hypersurface $\Pi$ of $\mathbb{S}_{1}^{4}$ defined as the intersection of $\mathbb{S}_{1}^{4}$ with the degenerate hyperplane $x_{1}=x_{5}$ of $\mathbb{R}_{1}^{5}$.

It is clear that $\mathbf{H}$ is lightlike if and only if $\Delta f+2 f$ is nowhere zero. On the other hand, an isometric immersion of a compact pseudo-umbilical spacelike surface $S$ into 
the De Sitter spacetime $\mathbb{S}_{1}^{4}$ with parallel lightlike mean curvature vector, is totally umbilical [19]. Moreover, if $S$ is a pseudo-umbilical surface in $\mathbb{S}_{1}^{4}$ with $g(\mathbf{H}, \mathbf{H})=0$, then it is easy to see that $\mathbf{H}$ is parallel if and only if $\mathbf{H}$ is a fixed vector of $\mathbb{R}_{1}^{5}$. Since an eigenfunction of $-\Delta$ corresponding to the eigenvalue -2 is the restriction to $\mathbb{S}^{2}$ of a homogeneous polynomial of degree $1,[20]$, we have that $\psi$ is totally umbilical if and only if $f$ is given by

$$
f(x, y, z)=a x+b y+c z+d,
$$

where $a, b, c, d \in \mathbb{R}$. Notice that there exist functions $f \in C^{\infty}\left(\mathbb{S}^{2}\right)$ such that $\mathbf{H}$ is a lightlike non-parallel vector field. In fact, it suffices to take $f(x, y, z)=x y-2$ and then we have $\mathbf{H}=-2(x y+1) \eta$. In such cases, $\mathbb{S}^{2}$ is a compact marginally trapped surface embedded in $\mathbb{S}_{1}^{4}$ and $\psi$ is a non-totally umbilical.

Example 5.3 Let $f: \mathbb{H}^{2} \rightarrow \mathbb{R}$ be a smooth function. Then, the isometric immersion $\psi: \mathbb{H}^{2} \rightarrow \mathbb{H}_{1}^{4}$ defined by

$$
\psi(x, y, z)=(f(x, y, z), x, y, z, f(x, y, z))
$$

is a 0 -isotropic MOTS in $\mathbb{H}_{1}^{4}$. In fact, the second fundamental form $h$ of $\psi$ is given by

$$
h(X, Y)=\left(\left(\nabla^{2} f\right)(X, Y)-f g(X, Y)\right) \eta,
$$

where $\nabla^{2} f$ is the Hessian of $f$ in $\mathbb{H}^{2}$ and $\eta$ denotes the lightlike vector $(1,0,0,0,1) \in$ $\mathbb{R}_{2}^{5}$. Thus, the immersion $\psi$ is 0 -isotropic with mean curvature vector

$$
\mathbf{H}=\frac{1}{2}(\Delta f-2 f) \eta
$$

Moreover, $\psi\left(\mathbb{H}^{2}\right)$ is contained in the degenerate totally geodesic hypersurface $\Pi$ of $\mathbb{H}_{1}^{4}$, defined as the intersection of $\mathbb{H}_{1}^{4}$ with the degenerate hyperplane $x_{1}=x_{5}$ of $\mathbb{R}_{2}^{5}$. Note that there exist functions $f \in C^{\infty}\left(\mathbb{H}^{2}\right)$ such that $\mathbf{H}$ is a non-parallel lightlike vector field. In fact, it suffices to take $f(x, y, z)=x^{2}+y^{2}$ and then $\mathbf{H}=2\left(x^{2}+y^{2}\right) \eta$. In such cases $\mathbb{H}^{2}$ is a non-totally umbilical isotropic marginally trapped surface embedded in $\mathbb{H}_{1}^{4}$.

For a 4-dimensional Lorentzian space form $M_{1}^{4}(c)$ of constant sectional curvature $c$ we have the following result.

Lemma 5.4 Let $\phi: S \rightarrow M_{1}^{4}(c)$ be a spacelike 0-isotropic immersion without flat points. Then, $\phi(S)$ is contained in a degenerate totally geodesic hypersurface $\Pi$ of $M_{1}^{4}(c)$. In particular, there exits a constant lightlike normal vector field $\eta$ such that $g(\phi, \eta)=0$ and $\mathbf{H}$ is proportional to $\eta$.

PROOF. From Corollary 4.4 we have that the first normal space $\operatorname{Im}(h)$ is a normal subbundle spanned by a lightlike vector at each point $p \in S$ and

$$
g(h(X, Y), h(Z, W))=0,
$$


holds for any $X, Y, Z, W \in \mathfrak{X}(S)$. Now, a simple differentiation of equation (5.1) with respect to any tangent vector field $T$ in $S$ gives

$$
g\left(\left(\bar{\nabla}_{T} h\right)(X, Y), h(Z, W)\right)=-g\left(h(X, Y),\left(\bar{\nabla}_{T} h\right)(Z, W)\right) .
$$

Combining repeatedly Codazzi's equation $\left(\bar{\nabla}_{X} h\right)(Y, Z)=\left(\bar{\nabla}_{Y} h\right)(X, Z)$ and equation (5.2), we find $g\left(\left(\bar{\nabla}_{T} h\right)(X, Y), h(Z, W)\right)=0$. Since the normal space is Lorentzian and two-dimensional we have $\left(\bar{\nabla}_{T} h\right)(X, Y) \in \operatorname{Im}(h)$ for any $X, Y, T \in \mathfrak{X}(S)$. This shows that the first normal space $\operatorname{Im}(h)$ is $D$-parallel. Therefore $\phi(S)$ is contained in a degenerate totally geodesic hypersurface $\Pi$ of $M_{1}^{4}(c)$ [21], and consequently, there exits a lightlike constant normal vector field $\eta$ such that $g(\phi, \eta)=0$ and $\mathbf{H}$ is proportional to $\eta$.

The flat points hypothesis in Lemma 5.4 is necessary as shown in the following example.

Example 5.5 Let $(x, y)$ be the canonical coordinates of $\mathbb{R}^{2}$ and consider the open set $S=\left\{(x, y) \in \mathbb{R}^{2}: 1<x^{2}+y^{2}<4\right\}$. Let $f: S \rightarrow \mathbb{R}$ be a smooth function such that $f>0$ on the sets

$$
U=\left\{(x, y) \in S: 2-\sqrt{1-x^{2}}<y<\sqrt{4-x^{2}}\right\}
$$

and

$$
V=\left\{(x, y) \in S:-\sqrt{4-x^{2}}<y<-2+\sqrt{1-x^{2}}\right\},
$$

while $f=0$ on $S-(U \cup V)$. Define $\phi: S \rightarrow \mathbb{R}_{1}^{4}$ as the isometric immersion given by

$$
\phi(x, y)= \begin{cases}(f(x, y), x, y, f(x, y)) & \text { if } y \geq 0 \\ (f(x, y), x, y,-f(x, y)) & \text { if } y<0\end{cases}
$$

Then $\phi$ is a spacelike 0 -isotropic immersion with flat points, which is full in $\mathbb{R}_{1}^{4}$, i. e., $\phi(S)$ is contained in no hyperplane of $\mathbb{R}_{1}^{4}$.

The following result determines complete spacelike 0 -isotropic surfaces of $\mathbb{R}_{1}^{4}$ without flat points.

Theorem 5.6 Let $\phi: S \rightarrow \mathbb{R}_{1}^{4}$ be a complete spacelike 0-isotropic immersion without flat points. Then,

(a) $S$ is isometric to $\mathbb{R}^{2}$.

(b) $\phi$ is congruent to an isometric immersion $\psi: \mathbb{R}^{2} \rightarrow \mathbb{R}_{1}^{4}$ given by

$$
\psi(x, y)=(f(x, y), x, y, f(x, y)),
$$

for some smooth function $f: \mathbb{R}^{2} \rightarrow \mathbb{R}$. Moreover, $S$ is a MOTS in $\mathbb{R}_{1}^{4}$ with mean curvature vector

$$
\mathbf{H}=\frac{1}{2} \Delta f \eta,
$$

where $\eta$ is a constant lightlike vector field of $\mathbb{R}_{1}^{4}$. 
PROOF. (a) From Lemma 5.4 the codimension can be reduced so that $\phi(S)$ is contained in a degenerate hyperplane $\Pi$ of $\mathbb{R}_{1}^{4}$ defined as the orthogonal complement of a constant lightlike vector field $\eta$ of $\mathbb{R}_{1}^{4}$. Now, consider a rigid motion $A$ of $\mathbb{R}_{1}^{4}$ sending $\eta$ (or a proportional vector to $\eta$ ) into $(1,0,0,1)$, and hence $\Pi$ onto the hyperplane $x_{1}=x_{4}$ of $\mathbb{R}_{1}^{4}$. Let $\pi: \mathbb{R}_{1}^{4} \rightarrow \mathbb{R}^{2}$ be the projection map onto the coordinates $\left(x_{2}, x_{3}\right)$. Then $\pi \circ A \circ \phi: S \rightarrow \mathbb{R}^{2}$ is an isometric immersion and, consequently, a local isometry. Now, by the completeness hypothesis, $\pi \circ A \circ \phi$ is an isometry [16, Corollaries 7.27, 7.29].

(b) As $\pi \circ A \circ \phi$ is an isometry, there exists a smooth function $f: \mathbb{R}^{2} \rightarrow \mathbb{R}$ such that $A$ sends $\phi(S)$ into the set of points $(f(x, y), x, y, f(x, y))$. Observe that the mean curvature vector of $A \circ \phi$ is given by $A(\mathbf{H})$. Now, from Example 5.1 we have $A(\mathbf{H})=\frac{1}{2} \Delta f(1,0,0,1)$ and the result follows.

From Proposition 4.1 and Theorem 5.6 we easily obtain the following ridigity results.

Corollary 5.7 Let $S$ be a complete spacelike surface of $\mathbb{R}_{1}^{4}$. Then, $S$ is an isotropic marginally trapped surface if and only if $S$ is congruent to a surface defined by $\psi(x, y)=(f(x, y), x, y, f(x, y))$, where $f: \mathbb{R}^{2} \rightarrow \mathbb{R}$ is a smooth function such that $\Delta f$ is nowhere zero.

Corollary 5.8 Let $S$ be a complete spacelike surface of $\mathbb{R}_{1}^{4}$. Then, $S$ is a pseudoumbilical marginally trapped surface if and only if $S$ is congruent to a surface defined by $\psi(x, y)=(f(x, y), x, y, f(x, y))$, where $f: \mathbb{R}^{2} \rightarrow \mathbb{R}$ is a smooth function such that $\Delta f$ is nowhere zero.

Remark 5.9 The last result completely determines the complete spacelike pseudoumbilical surfaces with lightlike mean curvature vector in $\mathbb{R}_{1}^{4}$. Compare this result with Theorem 4.1 of [14]. On the other hand, note that the marginally trapped surface $S$ of this Corollary is totally umbilical if and only if $f(x, y)=a\left(x^{2}+y^{2}\right)+$ $b x+c y+d$ with $a, b, c, d \in \mathbb{R}, a \neq 0$ (see Example 5.1).

Now, we prove a result analogous to Theorem 5.6 for complete spacelike 0isotropic surfaces of $\mathbb{S}_{1}^{4}$ without flat points.

Theorem 5.10 Let $\phi: S \rightarrow \mathbb{S}_{1}^{4}$ be a complete spacelike 0-isotropic immersion without flat points. Then,

(a) $S$ is isometric to $\mathbb{S}^{2}$.

(b) $\phi$ is congruent to an isometric immersion $\psi: \mathbb{S}^{2} \rightarrow \mathbb{S}_{1}^{4}$ given by

$$
\psi(x, y, z)=(f(x, y, z), x, y, z, f(x, y, z)),
$$

for some smooth function $f: \mathbb{S}^{2} \rightarrow \mathbb{R}$, and $S$ is a compact MOTS of $\mathbb{S}_{1}^{4}$ with mean curvature vector 


$$
\mathbf{H}=\frac{1}{2}(\Delta f+2 f) \eta
$$

where $\eta$ is a constant lightlike vector field of $\mathbb{R}_{1}^{5}$.

PROOF. (a) From Lemma $5.4 \phi(S)$ is contained in a degenerate hypersurface $\Pi$ of $\mathbb{S}_{1}^{4}$ defined as the intersection of $\mathbb{S}_{1}^{4}$ with the orthogonal complement of a constant lightlike vector field $\eta$ of $\mathbb{R}_{1}^{5}$. Now we consider a rigid motion $A$ of $\mathbb{S}_{1}^{4}$ such that $A$ (viewed as a rigid motion of $\left.\mathbb{R}_{1}^{5}\right)$ sends $\eta$ into $(1,0,0,0,1)$, and hence $\Pi$ onto the hyperplane $x_{1}=x_{5}$. Let $\pi: \mathbb{R}_{1}^{5} \rightarrow \mathbb{R}^{3}$ be the projection onto the coordinates $\left(x_{2}, x_{3}, x_{4}\right)$. Then $\pi \circ A \circ \phi: S \rightarrow \mathbb{S}^{2}$ is an isometric immersion and, by the completeness hypothesis, an isometry.

(b) As $\pi \circ A \circ \phi$ is an isometry, there exists a smooth function $f: \mathbb{S}^{2} \rightarrow \mathbb{R}$ such that $A$ sends $\phi(S)$ into the set of points $(f(x, y, z), x, y, z, f(x, y, z))$. Observe that the mean curvature vector of $A \circ \phi$ is given by $A(\mathbf{H})$. Now, from Example 5.2, $A(\mathbf{H})=\frac{1}{2}(\Delta f+2 f)(1,0,0,0,1)$ and the result follows.

By Proposition 4.1 and Theorem 5.10 we have also the following ridigity results.

Corollary 5.11 Let $S$ be a complete spacelike surface of $\mathbb{S}_{1}^{4}$. Then, $S$ is an isotropic marginally trapped surface if and only if $S$ is congruent to a surface defined by $\psi(x, y, z)=(f(x, y, z), x, y, z, f(x, y, z))$, where $f: \mathbb{S}^{2} \rightarrow \mathbb{R}$ is a smooth function such that $\Delta f+2 f$ is nowhere zero.

Corollary 5.12 Let $S$ be a complete spacelike surface of $\mathbb{S}_{1}^{4}$. Then, $S$ is a pseudoumbilical marginally trapped surface if and only if $S$ is congruent to a surface defined by $\psi(x, y, z)=(f(x, y, z), x, y, z, f(x, y, z))$, where $f: \mathbb{S}^{2} \rightarrow \mathbb{R}$ is a smooth function such that $\Delta f+2 f$ is nowhere zero.

We can also prove the corresponding $\mathbb{H}_{1}^{4}$-versions of Theorem 5.10 and Corollaries $5.11,5.12$ as follows.

Theorem 5.13 Let $\phi: S \rightarrow \mathbb{H}_{1}^{4}$ be a complete spacelike 0-isotropic immersion without flat points. Then,

(a) $S$ is isometric to $\mathbb{H}^{2}$.

(b) $\phi$ is congruent to an isometric immersion $\psi: \mathbb{H}^{2} \rightarrow \mathbb{H}_{1}^{4}$ given by

$$
\psi(x, y, z)=(f(x, y, z), x, y, z, f(x, y, z)),
$$

for some smooth function $f: \mathbb{H}^{2} \rightarrow \mathbb{R}$, and $S$ is a MOTS in $\mathbb{H}_{1}^{4}$ with mean curvature vector

$$
\mathbf{H}=\frac{1}{2}(\Delta f-2 f) \eta
$$

where $\eta$ is a constant lightlike vector field of $\mathbb{R}_{2}^{5}$. 
Corollary 5.14 Let $S$ be a complete spacelike surface of $\mathbb{H}_{1}^{4}$. Then, $S$ is an isotropic marginally trapped surface if and only if $S$ is congruent to a surface defined by $\psi(x, y, z)=(f(x, y, z), x, y, z, f(x, y, z))$, where $f: \mathbb{H}^{2} \rightarrow \mathbb{R}$ is a smooth function such that $\Delta f-2 f$ is nowhere zero.

Corollary 5.15 Let $S$ be a complete spacelike surface of $\mathbb{H}_{1}^{4}$. Then, $S$ is a pseudoumbilical marginally trapped surface if and only if $S$ is congruent to a surface defined by $\psi(x, y, z)=(f(x, y, z), x, y, z, f(x, y, z))$, where $f: \mathbb{H}^{2} \rightarrow \mathbb{R}$ is a smooth function such that $\Delta f-2 f$ is nowhere zero.

\section{Summary}

In this paper we have considered two families of surfaces in spacetimes: isotropic and marginally trapped surfaces. The notion of $\lambda$-isotropic surface means that the geometry of the surface is the same at each point regardless of direction, but may vary from point to point (unless $\lambda$ is constant). Then we have shown that the isotropy function $\lambda$ is the squared norm of the mean curvature vector field of the surface and also satisfies a key equation (Eq. (4.1)) involving the extrinsic geometry of the surface. As a consequence, we have seen that $\lambda \equiv 0$ is equivalent to the surface being extrinsically flat. On the other hand, we have developed a method to produce examples of isotropic MOTS in the classical models of spacetime: (i) for any differentiable function $f(x, y)$ on $\mathbb{R}^{2}$ we have given an example of 0 -isotropic MOTS in the Minkowski spacetime $\mathbb{R}_{1}^{4}$; (ii) for any differentiable $f(x, y, z)$ on the two-sphere $\mathbb{S}^{2}$ we have exhibited a 0-isotropic MOTS in the De Sitter spacetime $\mathbb{S}_{1}^{4}$; (iii) for any differentiable function $f(x, y, z)$ on the hyperbolic plane $\mathbb{H}^{2}$ we have shown a 0-isotropic MOTS in the anti De Sitter space $\mathbb{H}_{1}^{4}$. Furthermore, we have seen that it is possible to take an appropriate function $f$ such that the resulting embedded surface has a non-parallel null mean curvature vector field, and hence the surface is marginally trapped. It is worth pointing out that we have also provided ridigity results from these examples, that is, each of these examples serve as a model of 0-isotropic marginally trapped surfaces in the Minkowski, De Sitter and anti De Sitter spacetime, respectively. In fact, we have shown that any isotropic marginally trapped surface in $\mathbb{R}_{1}^{4}, \mathbb{S}_{1}^{4}$ or $\mathbb{H}_{1}^{4}$ is congruent to the surface given in example (i),(ii) or (iii), respectively.

\section{ACKNOWLEDGMENTS}

Research partially supported by OGICYT Grant No. MTM 2007-61284 and a PAY FQM-327 of the Junta de Andalucía(Spain)

\section{References}

[1] O’Neill B 1965 Canad. J. Math. 17907

[2] Kim Y 1995 Diff. Geom. Applic. 5321 
[3] Cabrerizo J L, Fernández M and Gómez J S 2009 J. Geom. Phys. 59834

[4] Cabrerizo J L, Fernández M and Gómez J S 2008 Taiwanese J. Math. 12 no.7 1707

[5] Penrose R 1965 Phys. Rev. Lett. 1457

[6] Bizon P, Malec E and Ò Muuchadha N 1988 Phys. Rev. Lett. 61 no.10 1147

[7] Pajerski D and Newman E 1971 J. Math. Phys. 121929

[8] Mars M and Senovilla J 2003 Class. Quamtum Grav. 20293

[9] Senovilla J 2002 Class. Quantum Grav. 19113

[10] Chen B 2009 Int. Electron. J. Geom. 2 no.1 1

[11] Andersson L, Mars M and Simon W 2008 Adv. Theor. Math. Phys. 12 no.4 853

[12] Schnetter E and Krishnan B 2006 Phys. Rev. D 73 021502(R)

[13] Senovilla J 2003 J. High Energy Phys., 11046

[14] Chen B Y and Van der Veken J 2007 Class. Quantum Grav. 24551

[15] Chen B Y and Van der Veken J 2007 J. Math. Phys. 48 no.7 073509

[16] O'Neill B 1983, Semi-Riemannian Geometry with Applications to Relativity (New York: Academic)

[17] Dajczer M 1990 Submanifolds and isometric immersions (Houston: Mathematics Lectures Series 13, Publish and Perish)

[18] Dajczer M and Fornari S 1987 Yokohama Math. J. 3561

[19] Alías L, Estudillo J and Romero A 1997 Tsukuba J. Math. 21169

[20] Berger M 1971 Le spectre d'une variété Riemannienne (Berlin: Lecture notes in Mathematics 194 Springer)

[21] Dajczer M 1984 Rev. Un. Mat. Arg. 31167 